\title{
Bacillus spores as building blocks for stimuli- responsive materials and nanogenerators
}

\section{Citation}

Chen, Xi, L. Mahadevan, Adam Driks, and Ozgur Sahin. 2014. “Bacillus Spores as Building Blocks for Stimuli-Responsive Materials and Nanogenerators." Nature Nanotechnology 9 (2) (January 26): 137-141. doi:10.1038/nnano.2013.290. http://dx.doi.org/10.1038/nnano.2013.290.

\section{Published Version}

doi:10.1038/nnano.2013.290

\section{Permanent link}

http://nrs.harvard.edu/urn-3:HUL.InstRepos:11857769

\section{Terms of Use}

This article was downloaded from Harvard University's DASH repository, and is made available under the terms and conditions applicable to Other Posted Material, as set forth at http:// nrs.harvard.edu/urn-3:HUL.InstRepos:dash.current.terms-of-use\#LAA

\section{Share Your Story}

The Harvard community has made this article openly available.

Please share how this access benefits you. Submit a story.

\section{Accessibility}




\title{
Spores of Bacillus as building blocks of high energy density stimuli-responsive materials
}

\author{
Xi Chen, L Mahadevan, Adam Driks \& Ozgur Sahin
}

Materials that respond mechanically to external chemical stimuli have wide-ranging applications in biomedical devices, adaptive architectural systems, robotics, and energy harvesting ${ }^{1}$. Synthesis and design principles inspired by biological systems have led to materials with capabilities for highly controlled and complex shape change ${ }^{2}$, oscillations ${ }^{3}$, fluid transport ${ }^{4}$, and homeostasis ${ }^{5}$. Despite the enhanced control over material behavior, the effectiveness of synthetic stimuli-responsive materials in generating work has been limited when compared to mechanical actuators ${ }^{6}$. Biological organisms with structures responsive to water gradients could potentially offer a solution for the limited work density of stimuli-responsive materials. Because water-responsive biological structures accomplish vital tasks like ascent of $\operatorname{sap}^{7,8}$, dispersal and self-burial of seeds ${ }^{9,10}$, they could possibly exhibit high energy densities and serve as building blocks of stimuli-responsive materials effective in generating work. Furthermore, biological nature of these materials offers the possibility of improving their characteristics through genetic mutations ${ }^{11,12}$. Here we report the discovery that the response of the spores of Bacillus to water potential gradients exhibit energy densities more than $10 \mathrm{MJ} / \mathrm{m}^{3}$, exceeding best synthetic waterresponsive materials by 1000 -fold ${ }^{13,14}$. We also identified a mutant spore form that nearly doubles the energy density relative to its wild type, highlighting the possibility for further improvements with genetic engineering of spores. We found that spores can self-assemble into dense, submicron-thick monolayers on substrates like silicon microcantilevers and elastomer sheets, creating bio-hybrid hygromorph actuators $^{15}$. The spore monolayers forming these hygromorphs exhibited high-energy density and rapid response to changing water potentials. As an application of the strong mechanical response of spores, we have built an energy harvesting device that can remotely generate electrical power from an evaporating body of water. These results demonstrate that spores have a significant potential as building blocks of stimuli-responsive materials with dramatically enhanced capabilities for energy harvesting, storage, and actuation of robotic devices.

Bacillus spores are dormant cells that can withstand harsh environmental conditions for long periods of time and still maintain biological functionality ${ }^{16}$ (Fig. 1a,b). Despite their dormancy, spores are dynamic structures. For example, Bacillus spores respond to changes in relative humidity (RH) by expanding and shrinking and changing their diameter by as much as $12 \%{ }^{17-19}$. We have used an atomic force microscope (AFM) based experiment (Fig. 2c) to determine the energy density of individual spores as they respond to changes in $\mathrm{RH}$. By adjusting force and $\mathrm{RH}$, we have created a thermodynamic cycle, in which individual spores go through four stages illustrated in Fig. 1d. In stage I, the spores rest at low RH ( 20\%). In stage II, the cantilever exerts a predetermined force (loading). In stage III, the spores are subjected to high $\mathrm{RH}(\sim 90 \%)$. In stage IV, the force is reduced back to zero. The cycle is completed upon lowering the $\mathrm{RH}$. 

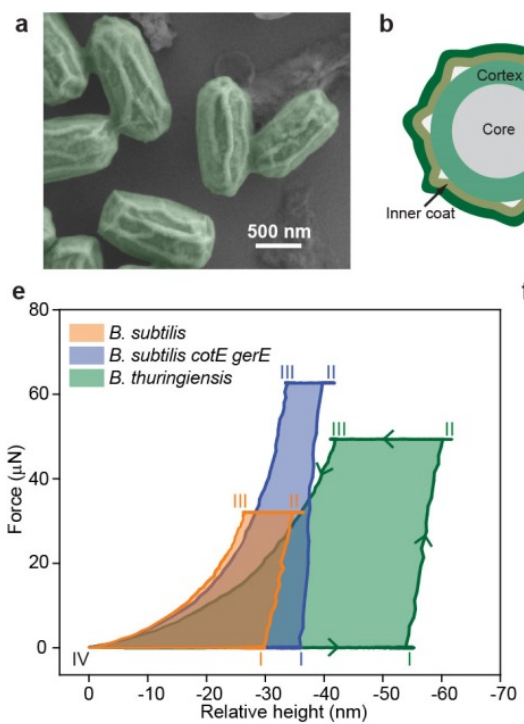

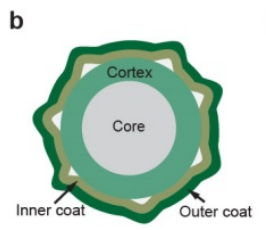

C

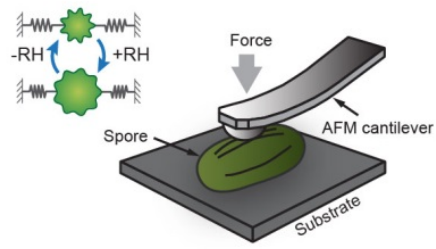

f

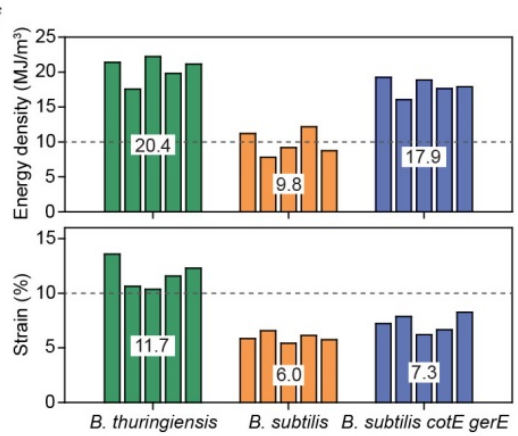

d

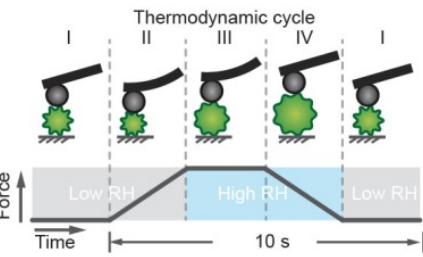

g



Figure 1. High energy density of Bacillus spores. (a) A false colored scanning electron microscope image of B. subtilis spores. (b) Spores are composed of multiple concentric shells encasing dehydrated genetic material at the center (the core). One of these shells is a loosely cross-linked peptidoglycan layer, called the cortex, which can absorb water and swell, causing coat to unfold ${ }^{18-20}$. (c) Expansion and contraction of spores in response to changing RH can potentially generate work that can be measured by AFM. (d) Illustration of a thermodynamic cycle created by controlling the force and relative humidity. (e) Force vs. relative-height curves during the thermodynamic cycle. (f) Energy densities and strain responses of five spores from different spore types. (g) Comparison of the results in ( $f$ ) with typical values reported for materials frequently used or studied in the context of actuators ${ }^{6}$, and stimuli-responsive materials ${ }^{13}$ (see supplementary information for additional references and numerical values used to create $(\mathrm{g}))$.

Figure 1e shows experimentally measured force vs. height curves as two wild type spores (B. subtilis and $B$. thuringiensis) and a mutant spore (B. subtilis cotE gerE) go through the thermodynamic cycle. The areas enclosed by the force vs. height curves in Fig. 1e correspond to the amounts of work done by the spores, which are on the order of $1 \mathrm{pJ}$. To estimate the energy density of spores, it is necessary to determine the volume of the material contributing to work. However, because stresses are localized near the apex of the tip, it is difficult to define the interaction volume. To account for the non-uniformity in stress and strain distributions, we approximated the spores with a cylindrical object (diameter $x$ length: $\sim 0.7 \times \sim 1.5 \mu \mathrm{m}$ ) indented by a spherical tip (radius: $\sim 0.85 \mu \mathrm{m}$ ). We estimated the energy density of spores by comparing the energy of indentation calculated for this geometry with the case where the spore is uniformly compressed by a planar indenter with the same depth of indentation (see supplementary information for details of the calculations). The resulting estimates of energy densities are plotted in Fig. If together with the maximum strains corresponding to the relative changes in spore diameters. 
We note that the spores studied here showed differences in both energy densities and strain responses. These differences can be partially explained by the relative size and swelling ability of the cortex layers in each species. For example, $B$. subtilis cotE gerE lacks most of its coat proteins, which reduces the overall volume of the spore, and therefore increase the relative cortex volume (see Fig. 1b). These effects may explain the increase in strain response and energy density of this mutant relative to its wild type. We also note that the maximum forces we could reversibly apply to the spores were consistently higher for the cotE gerE mutant than the wild type, which also contributed to the relative increase in energy density of these mutants. This favorable outcome suggests that an improved understanding of the hierarchical spore ultrastructure under extreme forces ${ }^{21}$ can lead to further increases in energy density and strain response through genetic engineering of spores.

The spores we studied here exhibited energy densities $\left(\sim 15 \mathrm{MJ} / \mathrm{m}^{3}\right)$ that are three orders of magnitude higher than synthetic water-responsive materials optimized for energy density ${ }^{13}\left(\sim 10 \mathrm{KJ} / \mathrm{m}^{3}\right)$. Because majority of the water absorption and release in spores is accomplished by the cortex, the energy density of the cortex material alone is likely to be even higher than the values for the entire spores. A simple estimate can be based on relative cortex volume, which could be obtained by approximating the coat and cortex layers as cylindrical shells encasing the core with respective thicknesses and radius of $50 \mathrm{~nm}$, $70 \mathrm{~nm}$, and $200 \mathrm{~nm}$, respectively. These numbers result in a cortex energy density of $\sim 60 \mathrm{MJ} / \mathrm{m}^{3}$. We also estimate the strain in cortex to be around $50 \%$ assuming majority of the diameter change is due to expansion of this layer. Figure $1 \mathrm{~g}$ compares the energy density and strain response of spores and the cortex with typical values reported for materials frequently used or studied in the context of actuators and stimuli-responsive materials. As seen in this comparison, spores are remarkable actuators with extreme characteristics.

The mechanical behavior individual spores suggest that they can potentially serve as building blocks of high-energy density stimuli-responsive materials. The small physical dimensions of spores allow using self-assembly to create approximate monolayers by depositing a suspension with a predetermined concentration of spores onto substrates and allowing it to dry (fig. 2a,b). With this approach, we assembled spores of $B$. subtilis on silicon microcantilevers and latex rubber sheets, and observed their response to changes in water potential. (The choice of species is influenced by its availability in large quantities and the lack of the exosporium layer in B. subtilis that could otherwise reduce the packing density on the surface). The differential strain exerted by the spores caused microcantilevers and latex sheets to change their curvatures, as illustrated in Fig. 2c,g. These deformations allow characterizing the response of the single-cell thick spore layer to changes in water potential. We determined the curvature of the spore-coated latex sheet from pictures taken by a camera. However, because of the small sizes of the microcantilevers, we used an AFM to characterize their deformations by reflecting the laser from the uncoated surface (Fig. 2d,e).

Measurements with the spore-coated microcantilever demonstrated excellent reversibility and a relatively fast response to variations in water potential. Figure $2 \mathrm{f}$ shows the response of the microcantilever (width $\times$ length $\times$ thickness: $30 \times 300 \times 1.5 \mu \mathrm{m})$ to cyclical variations in $\mathrm{RH}(15 \% \mathrm{vs.} 85 \%)$ before and after one million cycles. The data show that the spores initially moved the tip of the 
cantilever by $18 \mu \mathrm{m}$, which corresponds to a strain of $\sim 0.04 \%$. During this process, the spore layer generated a plane stress of $25.4 \mathrm{~N} / \mathrm{m}$. Following one million cycles of low and high $\mathrm{RH}$, the variations in the tip deflection and the corresponding surface stress reduced only slightly, demonstrating a high reversibility in the response of the spore monolayer. This reversibility is important for potential technological applications.

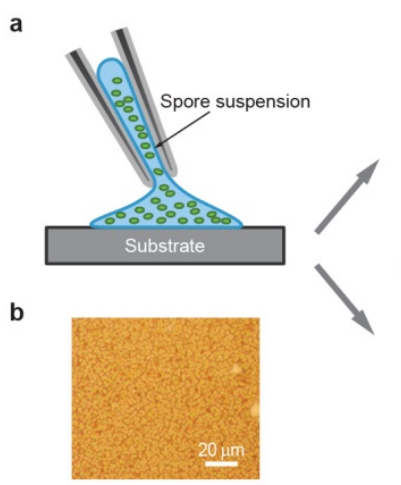

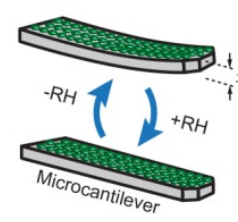

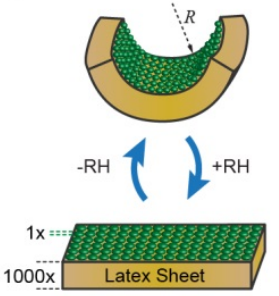

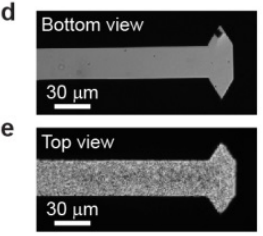

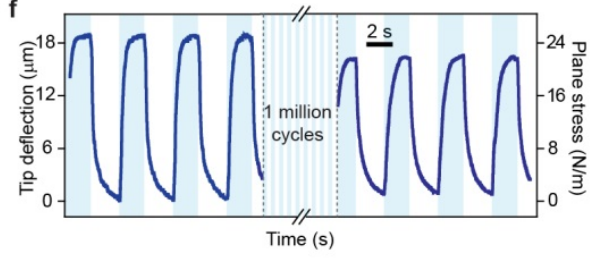

h
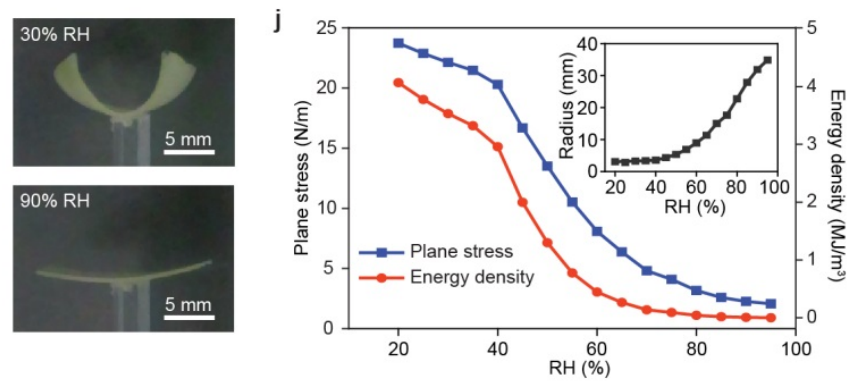

Figure. 2. Assembling spores into high energy density stimuli-responsive materials. By depositing a spore suspension onto a substrate (a) and allowing it to dry, a dense monolayer of spores is created (b). Spores placed on silicon microcantilevers (c) and latex rubber sheets (g) induce differential strain to cause curvature. Photographs of the (d) top and (e) bottom of a silicon AFM cantilever. ( $f$ ) The displacements of the tip of the cantilever in response to changing $\mathrm{RH}$, recorded before and after 1 million cycles of $\mathrm{RH}$ between $15 \%$ and $85 \%$. The corresponding surface stress was determined using Stoney's formula ${ }^{22}$. Photographs of the rubber sheet at $30 \%(\mathrm{~h})$ and $90 \% \mathrm{RH}$ (i). The sheet is viewed along its short dimension. (j) Measured radii of curvature (inset), corresponding plane stresses (squares) at the spore/rubber interface and energy density of the spore layer plotted against RH.

The data in Fig. $2 \mathrm{f}$ also show that the spores responded mechanically within $\sim 0.4$ seconds after exposure to humidity and within $\sim 0.5$ seconds during water release. This relatively fast response has important consequences for power output and it can be explained by the small thickness of the spore monolayer, where wetting and drying can both occur very fast. Quantitatively, both the classical Washburn law for wetting and diffusive evaporation for drying follow similar dynamical laws with the time for both scaling as $\tau \sim h^{2} / D$ with $h$ thickness of the sheet, and $D$ the diffusivity ${ }^{15}$. Because power depends on these time constants, thinner films have a considerable advantage. Power measurements that will be presented later provide experimental evidence for this characteristic.

The work done by the spores to bend the underlying flexible substrate can be maximized with a proper choice of substrate material and thickness. To understand the conditions that maximize energy output, 
we used a simple estimate for the maximum strain in a bilayer plate ${ }^{15,23}$. For a given ratio of the elastic modulus of the passive sheet to the spore layer, there is an optimum ratio of thicknesses that maximizes energy transfer (see supplementary online text). Based on this analysis, we prepared samples by placing spores on $0.5 \mathrm{~mm}$ thick latex rubber sheets, and allowed the sheets to deform in a horizontal plane to minimize the effect of gravity in our analysis.

Varying the level of $\mathrm{RH}$ produced dramatic changes in the shape of a $0.5 \mathrm{~mm}$ thick rubber sheet coated with a single-cell thick layer of bacterial spores (Fig. 3h,i). From the observed radii of curvature we determined the surface stress and the energy density of the spore monolayer. At $20 \% \mathrm{RH}$, the strain was $10.9 \%$ and the stress produced $(23.7 \mathrm{~N} / \mathrm{m})$ corresponds to a free energy of $\sim 2.6 \mathrm{~J} / \mathrm{m}^{2}$ and a work density of $\sim 4 \mathrm{MJ} / \mathrm{m}^{3}$ (assuming a thickness of $650 \mathrm{~nm}$ for the spore layer). This value is fairly close to the estimates based on AFM measurements of individual $B$. subtilis spores $\left(9.8 \mathrm{MJ} / \mathrm{m}^{3}\right)$, suggesting that the assembly process largely preserved the actuation ability of spores.

a Hygrovoltaic cell

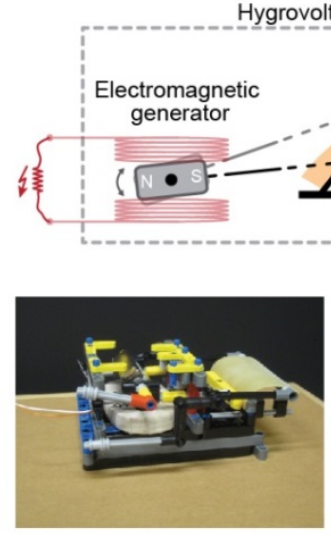

C


Figure 3. Application of spore based materials in energy harvesting: the hygrovoltaic generator. (a) Schematic of the hygrovoltaic device placed next to an open container filled with water. We used an electric fan to generate two streams of air - one flowing over the surface of the water and the other reaching directly to the device - and a baffle was placed $1.5 \mathrm{~cm}$ above the water surface to direct the moistened air towards the generator. As the air reaching to the device is switched between moist and dry, electrical energy is delivered to the load resistor. (b) Photograph of the device used in the experiments. One of the two coils is removed to expose the magnet. (c) Photograph of the spore-coated latex sheet used in the experiment (width $\times$ length $\times$ height: $5 \times 8 \times 0.075 \mathrm{~cm}$ ). (d) Measurements of the voltage across the load, (e) instantaneous power, and (f) the electrical energy delivered to the load. 
To demonstrate a potential application of spore based stimuli-responsive materials, we have created an energy harvesting device, the hygrovoltaic generator, which remotely generates electricity from evaporations of a standing body of water. For this, we coupled a spore-coated rubber sheet to an electromagnetic generator and placed the device next to an open container of water (Fig. 4a-c). When dry and moist streams of air were alternated, the spore-based hygromorph rotated the magnet back and forth, causing an alternating current to pass through the $300 \mathrm{KOhm}$ load resistor (Fig. 4d-f). During this process the spores delivered an average power of $\sim 0.7 \mu \mathrm{W}$, which is already comparable to vibrational energy harvesters ${ }^{24}$. Remarkably, however, only $\sim 3 \mathrm{mg}$ of bacterial spores generated this power. The corresponding specific electrical power of spores $\sim 233 \mathrm{~mW} / \mathrm{kg}$ exceeded demonstrations with synthetic polymers ${ }^{13}$ by nearly 4000 times. We attribute this result to the scaling behavior of wetting and drying kinetics with the reduced thickness of the high energy density water-responsive layer.

The estimates of energy densities presented in this work suggest that spores are likely to be the highest energy density materials for generating mechanical work. Together with their ability to self-assemble into functional structures, the unique ability of spores in generating work may lead to novel stimuliresponsive materials for a wide range of applications including harvesting and storage of energy, and robotic actuation. Furthermore, the availability of a wide range of species of Bacillus, and the possibility of controlling their response through mutations, as demonstrated here with B. subtilis cotE gerE, may lead to stimuli-responsive materials with genetically defined characteristics.

1 Stuart, M. A. C. et al. Emerging applications of stimuli-responsive polymer materials. Nat Mater 9, 101-113, (2010).

2 Sidorenko, A., Krupenkin, T., Taylor, A., Fratzl, P. \& Aizenberg, J. Reversible switching of hydrogel-actuated nanostructures into complex micropatterns. Science 315, 487-490, (2007).

3 Yashin, V. V. \& Balazs, A. C. Pattern Formation and Shape Changes in Self-Oscillating Polymer Gels. Science 314, 798-801, (2006).

4 Wheeler, T. D. \& Stroock, A. D. The transpiration of water at negative pressures in a synthetic tree. Nature 455, 208-212, (2008).

$5 \mathrm{He}, \mathrm{X}$. et al. Synthetic homeostatic materials with chemo-mechano-chemical self-regulation. Nature 487, 214-218, (2012).

6 Madden, J. D. W. et al. Artificial muscle technology: Physical principles and naval prospects. IEEE J. Ocean. Eng. 29, 706-728, (2004).

$7 \quad$ Pickard, W. F. The ascent of sap in plants. Prog. Biophys. Mol. Biol. 37, 181-229, (1981).

8 Hacke, U. G., Sperry, J. S., Pockman, W. T., Davis, S. D. \& McCulloh, K. A. Trends in wood density and structure are linked to prevention of xylem implosion by negative pressure. Oecologia 126, 457-461, (2001).

9 Dawson, J., Vincent, J. F. V. \& Rocca, A. M. How pine cones open. Nature 390, 668-668, (1997).

10 Elbaum, R., Zaltzman, L., Burgert, I. \& Fratzl, P. The role of wheat awns in the seed dispersal unit. Science 316, 884-886, (2007).

11 Whaley, S. R., English, D. S., Hu, E. L., Barbara, P. F. \& Belcher, A. M. Selection of peptides with semiconductor binding specificity for directed nanocrystal assembly. Nature 405, 665-668, (2000).

12 Lee, B. Y. et al. Virus-based piezoelectric energy generation. Nature Nanotechnology 7, 351-356, (2012). 
13 Ma, M., Guo, L., Anderson, D. G. \& Langer, R. Bio-Inspired Polymer Composite Actuator and Generator Driven by Water Gradients. Science 339, 186-189, (2013).

14 Kim, H. \& Kwon, S. Water-Responsive Polymer Composites on the Move. Science 339, 150-151, (2013).

15 Reyssat, E. \& Mahadevan, L. Hygromorphs: from pine cones to biomimetic bilayers. J. R. Soc. Interface 6, 951-957, (2009).

16 Nicholson, W. L., Munakata, N., Horneck, G., Melosh, H. J. \& Setlow, P. Resistance of Bacillus endospores to extreme terrestrial and extraterrestrial environments. Microbiology and Molecular Biology Reviews 64, 548-572, (2000).

17 Westphal, A. J., Price, P. B., Leighton, T. J. \& Wheeler, K. E. Kinetics of size changes of individual Bacillus thuringiensis spores in response to changes in relative humidity. Proc. Natl. Acad. Sci. USA 100, 3461-3466, (2003).

18 Driks, A. The dynamic spore. Proc. Natl. Acad. Sci. USA 100, 3007-3009., (2003).

19 Plomp, M., Leighton, T., Wheeler, K. E. \& Malkin, A. J. The high-resolution architecture and structural dynamics of Bacillus spores. Biophys. J. 88, 603-608, (2004).

20 Sahin, O., Yong, E. H., Driks, A. \& Mahadevan, L. Physical basis for the adaptive flexibility of Bacillus spore coats. J. R. Soc. Interface 9, 3156-3160, (2012).

21 Buehler, M. J. \& Yung, Y. C. Deformation and failure of protein materials in physiologically extreme conditions and disease. Nature materials 8, 175-188, (2009).

22 Stoney, G. G. The tension of metallic films deposited by electrolysis. Proc. R. Soc. London, Ser. A 82, 172-175, (1909).

23 Timoshenko, S. Analysis of bi-metal thermostats. J. Opt. Soc. Am. Rev. Sci. Instrum. 11, 233-255, (1925).

24 Paradiso, J. A. \& Starner, T. Energy scavenging for mobile and wireless electronics. Pervasive Computing, IEEE 4, 18-27, (2005).

Acknowledgements: We acknowledge financial support from the Wyss Institute for Biologically Inspired Engineering, the Rowland Junior Fellows Program, and the Early Career research Program award. We thank to Donald E. Ingber for encouragement and comments on the manuscript, Chris P. Stokes for his help with the experimental setup for rapidly switching the humidity levels surrounding the spores, David Bell for his help with electron microscopy, John M. Sungur for prototype device manufacturing.

Author contributions: X.C. performed experiments in Figure 1 and analyzed the data; L.M. contributed to the theory and scaling estimates; A.D. contributed mutant and wild type spore samples. O.S. conceived and designed the experiments, performed experiments in Figure 2 and 3; O.S. and L.M. wrote the paper.

Author Information Correspondence and requests for materials should be addressed to O. S. (sahin@columbia.edu) 


\section{Methods:}

Experimental setup for rapid switching of RH: A laboratory air source was used to supply two streams of air travelling through plastic tubing. One of the streams was saturated with water vapor by passing it through a bubbler. The other stream carried the air at approximately 10 $20 \%$ relative humidity at the room temperature $\left(\sim 2{ }^{\circ} \mathrm{C}\right)$. A solenoid valve (SMC, NVFS) and a motorized arm were used to block the air from the tubes one at a time, switching at the frequency of a square wave supplied by a power MOSFET (NTD4960N). The drifts in height measurements due to possible mismatch in temperatures of dry and humid air were minimized ( $4 \mathrm{~nm}$ peak to peak) by focusing the air flow to a small region surrounding the cantilever. We also found that the relative humidity near the imaging area was affected by the temperature of the AFM head and the piezo-scanner, which are slightly above the room temperature. We placed an electric fan behind the AFM head to reduce the temperature around the imaging area down to $\sim 23{ }^{\circ} \mathrm{C}$.

AFM Indentation of single spores: A commercial AFM (Multimode SPM, Nanoscope V controller and Signal access module, Veeco Instruments) was used for AFM experiments. The indentation depth was passively monitored with Labview (National Instruments) using a data acquisition card (NI-DAQ S-series 6115). Forces applied by the cantilever were controlled by offsetting the feedback set-point value of the AFM with an analog summing amplifier (SIM 980, Stanford Research Systems). We used cantilevers with large tip radii $\sim 850 \mathrm{~nm}$, measured by SEM, and large spring constants $\sim 500 \mathrm{~N} / \mathrm{m}$ (LTRH probes, Team Nanotec). Spring constants were calibrated against reference cantilevers (Applied Nanostructures, FCL probes) that are separately calibrated using the Sader method. Spores were first inspected by tapping mode imaging using the same probes for possible contaminants and presence of exosporium (in the case of $B$. thuringiensis). Selected spores were indented in contact mode by controlling the feedback set-point value. We recorded cantilever deflection and vertical position signals with the data acquisition card. The work outputs of spores were maximized by adjusting the indentation speed and the duration of the humid state (stages II $\rightarrow$ III).

Coating the AFM cantilever with spores: An AFM cantilever chip (Veeco Instruments, HMX-S) was gently placed on a flat piece of silicon wafer while the cantilever stayed in contact with the surface of the wafer. A suspension of spores ( $1 \mathrm{~mm}$ in diameter) in water was pipetted onto the cantilever under an optical microscope and allowed to dry. The cantilever surface was inspected by optical microscopy to ensure spore coverage of its surface.

Measurement of changes in plane stress and mass of the cantilever: The plane stress $\sigma$ at the surface of an elastic substrate with thickness $t$, Young's modulus $E$, and Poisson's coefficient $v$ is related to the radius of curvature $r$ according to Stoney's formula ${ }^{22}$ : 


$$
\sigma=\frac{E}{6(1-v)} \frac{t^{2}}{r}
$$

This formula provides a good approximation if the film generating stress is significantly thinner than the substrate so that the bending stiffness of the film is negligible. While the thickness of the spore layer is comparable to the cantilever, we assumed that it has a negligible bending stiffness because it is composed of objects with approximately circular cross sections. A more general treatment of curvature can be found in ref. [15]. The AFM allows measurement of the slope of the cantilever at the location of the laser spot, rather than measurement of the radius of curvature. However, $r$ can be related to the slope $\theta$ if the position of the laser spot relative to the cantilever base $x$ is known. Assuming a parabolic profile for the cantilever, the radius is: $r=x / \theta$. The changes in the slope near the free end of the cantilever were measured to be well beyond the detector limits (saturation); therefore we placed the laser spot close to the cantilever base. The exact position of the laser spot was estimated by comparing the ratio of the thermal noise levels at this location and at a location near the free end of the cantilever, using the analytical expression for the mode shape of a rectangular cantilever beam. Thickness of the cantilever was determined to be 1.49 um from the spring constant. We used $E \sim 130 \mathrm{GPa}$ and $v \sim 0.278$ for silicon (100). We neglected the effect of the reflective aluminum coating (40 $\mathrm{nm}$ ) and assumed the entire cantilever is made of silicon.

Coating latex rubber sheets with spores: Natural latex rubber sheets (Rubber Sheet Roll, Amazon.com) were cut into rectangular pieces with scissors. Their top surfaces are treated with poly-I-lysine to improve adhesion. A solution containing $B$. subtilis spores was placed on pieces of rubber sheet and then allowed to dry in a fume hood. RH of the laboratory air was approximately $15-20 \%$. The amount of solution to be placed on the rubber sheet was determined by visually inspecting spore coverage under an optical microscope. Once the solution dried, the rubber sheets already exhibited a curvature because the RH of laboratory air was low ( 15-20\%). The sheets were then placed in a chamber with saturated air and kept for a day. This procedure increased the curvature of the rubber sheets once they were placed back to low $\mathrm{RH}$.

Measurement of plane stress and radius of curvature of the rubber sheet: The rubber sheet was cut into a $2 \mathrm{~cm}$ by $6 \mathrm{~cm}$ rectangular piece and coated with a layer of spores. The sheet was attached from the center to a piece of acrylic glass with adhesive tape and then placed vertically in a humidity chamber with transparent walls. $\mathrm{RH}$ inside the chamber was monitored with a hygrometer (Vaisala). The chamber RH was gradually increased from the laboratory level ( $18 \%$ at the time of the measurements) by supplying saturated air. Photographs of the latex sheet were taken from a direction allowing the $2 \mathrm{~cm}$ wide edge to be seen. Pictures were taken at intervals of $5 \% \mathrm{RH}$ starting from $20 \%$. 
The plane stress at the spore layer was determined according to the formula:

$$
\sigma_{x}=\frac{E t^{2}}{6\left(1-v^{2}\right)}\left(\frac{1}{R_{x}}-v \frac{1}{R_{y}}\right) .
$$

Here $\sigma_{\mathrm{x}}$ is the surface stress along the direction of the observed curvature, $E$ is the Young's modulus of the rubber, $v$ is the Poisson's coefficient for rubber, $t$ is the thickness of the rubber sheet, and $R_{x}, R_{y}$ are the radii of the curvature. $t$ is $0.5 \mathrm{~mm}$ for the sheet used in Fig. $3 . R_{x}$ is estimated by fitting the optical pictures of the rubber with a circle. $R_{y}$ is assumed to be infinite because the rubber sheet exhibited a cylindrical shape. Strain at the surface of the rubber sheet near the spores is estimated from $2 t / 3 R_{x}$ (the neutral plane is $2 t / 3$ below the surface, see also Eqs. 2.3-2.6 of ref[15]. We determined $E$ from the stress strain curves for a rectangular strip of the same latex rubber sample (1.3 MPa). $v$ is taken as 0.5 . Note that in contrast to Eq. (1), Eq. (2) accounts for anisotropic stresses in the spore layer. The cylindrical geometry of the rubber sheet originated during the spore drying. This shape was stable.

Experimental setup for electricity generation: A $0.750 \mathrm{~mm}$ thick latex rubber sheet, $5 \mathrm{~cm}$ by 8 $\mathrm{cm}$ in size, was coated with a layer of $B$. subtilis spores. The sheet was brought into physical contact with one end of a lever that pivots around a central axis like a seesaw. We connected the opposite end of the lever to an electromagnetic generator with a short string. The electromagnetic generator consisted of a stack of magnets rotating between two copper coils. Copper coils are formed by winding a total of $22 \mathrm{Kfeet}$ of 42 gauge magnet wire (Polytech Coil Winding, Tacoma, WA, USA). The magnet stack was composed of two $2^{\prime \prime} \times 1 / 2$ " $\times 1 / 4$ " and two 2 " $\times 1 / 2$ " $\times 1 / 8$ " neodymium magnets. We used silicon nitride ball bearings to allow magnets rotate with respect to the coils.

The container used to hold water was $9 \mathrm{~cm}$ wide along the direction parallel to the $8 \mathrm{~cm}$ long edge of the spore coated latex sheet. The length and depth of the container was $15 \mathrm{~cm}$ and 4 $\mathrm{cm}$ respectively. An aluminum foil was placed $1.5 \mathrm{~cm}$ above the water filled container so that water vapor can leave only from the $9 \mathrm{~cm}$ wide sides as illustrated in Fig. 3a. The container was placed on a ceramic-top heater (Corning $\left.{ }^{\circledR}, \mathrm{PC}-600 \mathrm{D}\right)$. The temperature of the water was monitored with a digital thermometer and kept at $31{ }^{\circ} \mathrm{C}$. The room temperature and $\mathrm{RH}$ were approximately $24{ }^{\circ} \mathrm{C}$ and $30 \%$, respectively. We used a low power DC electric fan to create air flow. The fan was placed either close to the $1.5 \mathrm{~cm}$ wide opening of the water container to transport moisture to the device or away from the opening to transport dry air to the device. A hot wire anemometer (Cole-Palmer, EW-30005-85) measured the air velocity in the vicinity of the latex rubber sheet to be less than $0.5 \mathrm{~m} / \mathrm{sec}$ in either position of the electric fan. Measurements with an empty container showed that the background power induced by the air flow was around $3.5 \mathrm{nW}$. 